\title{
DOWNLINK INTERCELL INTERFERENCE CANCELLATION IN WCDMA BY EXPLOITING EXCESS CODES
}

\author{
Massimiliano Lenardi, Abdelkader Medles and Dirk T.M. Slock \\ Mobile Communications Department - Institut Eurécom \\ 2229 Route des Crêtes, B.P. 193 \\ F-06904 Sophia Antipolis Cedex, FRANCE \\ $\{$ lenardi, medles, slock\}@eurecom.fr
}

\begin{abstract}
The deployment of third generation CDMA-based wireless systems foresees a loading fraction that is smaller than one, i.e. the number of users per cell is scheduled to be significantly less than the spreading factor to attain an acceptable performance. This means that a base station can set apart a subset of the codes, the excess codes, that it will not use. In the case of periodic codes (such as in the TDD mode of UMTS), the existence of excess codes implies the existence of a noise subspace, which can be used to cancel the interference coming from a neighboring base station. We introduce a SINR maximizing linear receiver to perform this interference reduction. In the case of aperiodic codes (such as in the FDD mode of UMTS), the noise subspace is time-varying. In this case, we introduce structured receivers that combine scrambling and descrambling operations with projections on code subspaces and linear time-invariant filters for equalization, interference cancellation and multipath combining. So the timevarying part of these receivers is limited to (de-)scrambling operations. The design of the various filter parts gets discussed. Performance improvements are illustrated via simulations.
\end{abstract}

\section{DOWNLINK SIGNAL MODEL}

Fig. 1 shows the downlink signal model in baseband. For each base station $j, j=1,2$, the $K^{j}$ users are assumed to transmit linearly modulated signals over the same linear multipath channel $h^{j}(t)$; the BS 2 is considered the interferring one. Additive noise $v(t)$ is then included in the received signal $y(t)$.

The symbol and chip periods $T$ and $T_{c}$ are related through the spreading factor $L: T=L T_{c}$, which is assumed here to be

Eurécom's research is partially supported by its industrial partners: Ascom, Swisscom, Thomson-CSF, IBM France, CEGETEL, Motorola, France Télécom, Hitachi Europe and Texas Instruments. The work leading to this paper was also partially supported by the French RNRT (National Network for Telecommunications Research) project AUBE. common for all the users and for the two base stations. The total chip sequences $b_{l}^{j}$ are the sum of the chip sequences of all the users for each BS $j$. Every user chip sequence is given by the product between the $n$th symbol of the $k$ th user and an aperiodic spreading sequence $w_{k, l}^{j}$ which is itself the product of a periodic Walsh-Hadamard (with unit energy) spreading sequence $\boldsymbol{c}_{k}^{j}=\left[\boldsymbol{c}_{k, 0}^{j} \boldsymbol{c}_{k, 1}^{j} \cdots c_{k, L-1}^{j}\right]^{T}$, and a base-station specific unit magnitude complex scrambling sequence $s_{l}^{j}$ with variance $1, w_{k, l}^{j}=c_{k, l \bmod L}^{j} s_{l}^{j}$ :

$$
b_{l}^{j}=\sum_{k=1}^{K^{j}} b_{k, l}^{j}=\sum_{k=1}^{K^{j}} a_{k,\left\lfloor\frac{l}{L}\right\rfloor}^{j} w_{k, l}^{j} \quad j=1,2 .
$$

The scrambling operation is a multiplication of chip rate

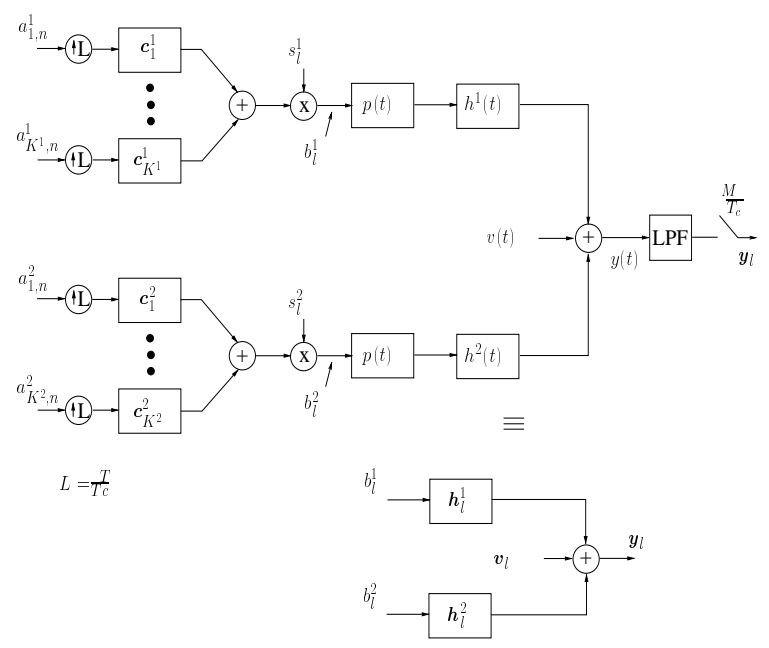

Figure 1: Downlink signal model

sequences. The spreading operation is represented by a filtering of an upsampled symbol sequence with the spreading sequence as impulse response. The chip sequences $b_{l}^{1,2}$ get transformed into a continuous-time signals by filtering them with the pulse shape $p(t)$ and then pass through the multipath propagation channels $h^{1}(t)$ and $h^{2}(t)$ (from BS 1 and 
from BS 2 to the mobile station respectively) to yield the total received signal $y(t)$. The receiver samples $M$ times per chip the lowpass filtered received signal. Stacking the $M$ samples per chip period in vectors, we get for the sampled received signal

$\boldsymbol{y}_{l}=\boldsymbol{y}_{l}^{1}+\boldsymbol{y}_{l}^{2}+\boldsymbol{v}_{l}, \quad \boldsymbol{y}_{l}^{j}=\sum_{k=1}^{K^{j}} \sum_{i=0}^{N-1} \boldsymbol{h}_{i}^{j} b_{k, l-i}^{j} \quad j=1,2$

where

$$
\boldsymbol{y}_{l}^{j}=\left[\begin{array}{c}
y_{1, l}^{j} \\
\vdots \\
y_{M, l}^{j}
\end{array}\right], \boldsymbol{h}_{l}^{j}=\left[\begin{array}{c}
h_{1, l}^{j} \\
\vdots \\
h_{M, l}^{j}
\end{array}\right], \boldsymbol{v}_{l}=\left[\begin{array}{c}
v_{1, l} \\
\vdots \\
v_{M, l}
\end{array}\right]
$$

Here $\boldsymbol{h}_{l}^{j}$ represents the vectorized samples of the overall channel $h^{j}(t)$, including pulse shape, propagation channel and receiver filter. The overall channels $h^{j}(t)$ are assumed to have the same delay spread of $N$ chips.

In the case where the scrambling sequence is aperiodic and where we model it and the symbol sequences as independent i.i.d. sequences, then the chip sequences $b_{l}^{1,2}$ are sums of independent white noises (chip rate i.i.d. sequences, hence stationary) and hence are white noises. The intracell contribution to $\boldsymbol{y}_{l}$ then is a stationary (vector) process (the continuous-time counterpart is cyclostationary with chip period). The intercell interference is a sum of contributions that are of the same form as the intracell contribution so its contribution to $\boldsymbol{y}_{l}$ is also a stationary vector process. The remaining noise is assumed to be white stationary noise. Hence the sum of intercell interference and noise, $\boldsymbol{v}_{l}$, is stationary.

When the scrambling sequence is periodic with (symbol) period $T$, the chip sequences $b_{l}^{1,2}$ are stationary (vector) processes with symbol period; the intracell contribution to $\boldsymbol{y}_{l}$ is then also stationary with symbol period. The intercell contribution is again of the same form of the intracell interference (while the noise stays white stationary), so that the received signal is cyclostationary with symbol period.

\section{RECEIVER STRUCTURES FOR INTERCELL INTERFERENCE CANCELLATION}

In this section we focus on the description of the receiver structures that we analyzed and implemented.

\subsection{Linear Structures}

Fig. 2 shows a linear receiver in which a descrambler and a correlator follow a general FIR filter $f_{l}$ whose input is at sampling rate and its output is at chip rate. In the case of a RAKE receiver implementation, $f$ is a channel matched

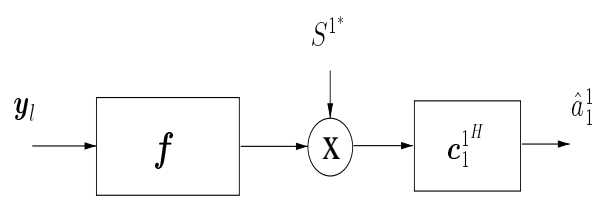

Figure 2: General Linear Receiver

filter $\boldsymbol{h}^{1^{H}}$ which is equivalent to consider the BS 2 as an additive noise and to maximize the SNR at the filter output. To maximize the SINR at the receiver output while taking into account the intracell interference, the filter $f$ can be adapted to be the Max-SINR filter (see [1]). These two approaches are well suitable for a system with one BS, but they are here used for comparison with other structures designed to take better into account the presence of an interferring base station (see 2.2).

\subsection{Hybrid Structures}

Fig. 3 shows one instance of an hybrid structure where an Interference Cancellation (IC) Branch preceeds a linear receiver as in Fig. 2. The IC branch is formed by an equalizer, a descrambler, a projector, a scrambler and a filter; different implementation are possible and are presented in the following section 2.2.1 to 2.2.3. This approach is conceived to perform intracell or intercell interference cancellation in order to improve the performances of the linear receivers presented above by exploiting the structure of the received signal itself (the IC branch is indeed equivalent to a timevariant filter).

\subsubsection{Structure 1}

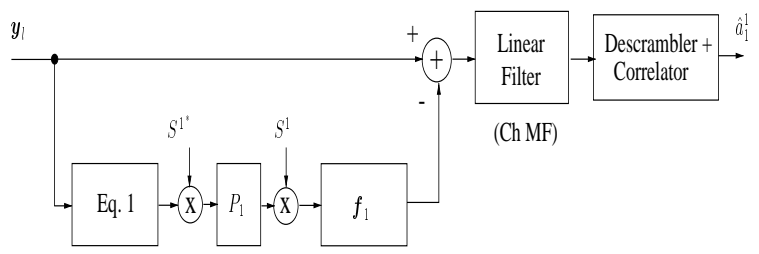

Figure 3: Hybrid Structure 1, intracell IC

Here the base station 1 is considered in the IC branch and intracell interference cancellation is performed by reconstructing the orthogonality between user codes of BS 1 after the equalizer and projecting this signal into the subspace of the used spreading codes, but the user of interest. Then, by "re-channeling" the scrambler output, the intracell interference can be subtructed from the received signal $\boldsymbol{y}_{l}$ and is possible to apply a RAKE receiver. This structure allows also to decrease the intercell interference plus noise because 
the projector output space include both of them. The equalizer could be either a Zero-Forcing (ZF) or a Minimun Mean Square Error (MMSE) equalizer: the former enhances more the intercell interference plus noise but restores the orthogonality, while the latter perturbs the structure of the signal received from the BS 1 but does not increase so much the intercell interference plus noise. The "re-channeling" filter could be either the channel $\boldsymbol{h}^{1}$ or a filter adapted to minimize the intercell interference plus noise variance. We can guess then when a ZF equalizer is used, this adaptation works better then in using a MMSE equalizer because in the first case the intercell interference plus noise is more important.

\subsubsection{Structure 2}

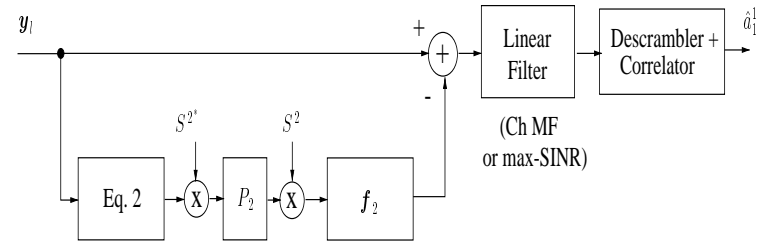

Figure 4: Hybrid Structure 2, intercell IC

In this structure, the BS 2 is considered in the IC branch; the equalizer and the "re-channeling" filter can again be described as in section 2.2.1, but the projection is into the complete subspace of used spreading codes of BS 2. In this approach is possible to implement either a RAKE receiver or a max-SINR receiver after the subtractor, being the latter conceivable due to the absence of the signal from BS 2 in the subtractor output signal.

\subsubsection{Structure 3}

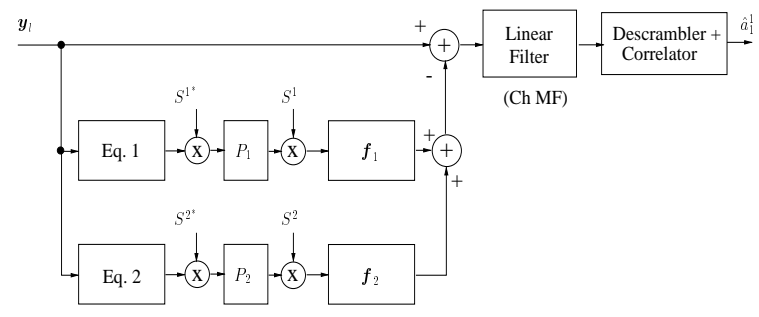

Figure 5: Hybrid Structure with two IC branches

This structure combines the single-branch approaches of sections 2.2.1 and 2.2.2. The equalizers and the projections are defined as before, while the two filters $\boldsymbol{f}_{1}$ and $\boldsymbol{f}_{2}$ can be equal to the corresponding BS channels $\boldsymbol{h}^{1}$ and $\boldsymbol{h}^{2}$ or jointly adapted to have minimum interference plus noise variance at the subtractor output. The linear receiver after the subtractor can be in this case just a RAKE receiver, due to the simultaneous cancellation of intracell and intercell interference.

\section{NUMERICAL EXAMPLES}

Fig. 6 to Fig. 14 present some of the simulations that we have performed to evaluate the various structures. In the legends of these figures, MS refer to max-SINR receiver. The $K^{j}$ users of base station $j$ are considered synchronous between them and with the users of the other BS, with the same spreading factor $L=32$ and using the same downlink channel $\boldsymbol{h}^{j}$ which is a FIR filter, convolution of a sparse Vehicular A UMTS channel and a pulse shape (root-raised cosine with roll-off factor of 0.22 ). The channel length is $N=$ 19 chips, due to the UMTS chip rate of $3.84 \mathrm{Mchips} / \mathrm{sec}$. An oversampling factor of $M=2$ is assumed. Two possible user power distributions are simulated: all interferers (intracell and intercell) have the same power and the user of interest has either the same power also or $10 \mathrm{~dB}$ less power (near-far situation). Two possible base-station dependent scrambling sequences are simulated: aperiodic and periodic (with symbol period) scrambling. Every simulation gives 10 curves: the first two in the legend are the ones described in section 2.1, the simple RAKE and the max-SINR receivers; the third to the sixth in the legend are referring to the structures in which the "re-channeling" filters are the channels themself, $\boldsymbol{f}_{j}=\boldsymbol{h}^{j} j=1,2$, while the last four in the legend are the same structures but with $f_{j}$ optimize as explained in section 2.2.1. The names in the legend "Si-X-RAKE" or "Si$\mathrm{x}$-MS" refer to the structure $\mathrm{i}$ with "re-channeling" filter $\mathrm{x}$ (channel or optimized filter) and output linear filter RAKE or max-SINR. In the case of structure 3 (section 2.2.3) $\mathrm{H}$ refers to the use of the two channels $\boldsymbol{h}^{1}$ and $\boldsymbol{h}^{2}$ and $\mathrm{F}$ to the use of the jointly optimized filters $f_{1}$ and $f_{2}$.

The first two figures, Fig. 6 and Fig. 7, show the performances in implementing respectively a $\mathrm{ZF}$ equalizer or a MMSE equalizer in the IC branches. We can well see how the MMSE versions work much better than the ZF ones. In Fig. 7, all the curves are above the simple RAKE curve. Another remark is that when a MMSE equalizer is implemented, the optimization of the filters $f_{j}$ is practically not necessary, which is not the case of ZF equalizers (as we stated in section 2.2.1). So the following figures for aperiodic scrambling are always supposing the use of MMSE equalizers and in this case the hybrid structure 3 works always much better than all the other structures. Fig. 8 confirms even more this result showing the case of near-far situation for the user of interest; the hybrid structure 3 is the best one on all the range of interest for $\mathrm{Eb} / \mathrm{No}$.

Fig. 9 and Fig. 10 show that all the structures are sensitive to the number of users in the system, but again the structure 3 is better because is less sensitive, expecially in the case of optimization of the "re-channeling" filters. 
Fig. 11 to Fig. 14 present the case when the scrambling for the two base stations is periodic with symbol period. In all simulations the simple max-SINR receiver (section 2.1 and [1]) is performing the best for all Eb/No. All the hybrid solutions show a saturation for high $\mathrm{Eb} / \mathrm{No}$, but the optimized (in terms of $\boldsymbol{f}_{j}$ ) hybrid structures 3 and 2 seem to perform better than the other hybrid structures. In these simulations we used ZF equalizations in the IC branches because a MMSE equalization is here (periodic scrambling) not conceivable, due to the fact that the signals are not anymore cyclostationary with chip period but they are cyclostationary with symbol period.

\section{CONCLUSIONS}

In the case of aperiodic scrambling, the classical linear receivers have some limitations when an interfering base station is present. At the same time the hybrid structures with a MMSE equalizer design show better performances, in particular the hybrid structure 3. The optimization of the "rechanneling" filters not being necessary when MMSE equalizers are used, the complexity of the structure is limited, even more so because MMSE equalizers can be implemented with LMS-style algorithms.

On the contrary, in the case of periodic scrambling, the max-SINR linear receiver outperforms all the other (linear and non-linear) structures. This is due to the fact that the excess codes (codes not used by a base station) give rise to time-invariant noise subspaces which are used to cancel the interfering base station. In the case of aperiodic scrambling, these noise subspaces are time-variant and the only way to exploit them for IC with time-invariant filters is with hybrid structures, such as the ones in section 2.2.

\section{REFERENCES}

[1] L. Massimiliano and D. T. M. Slock, "A RAKE Receiver with Intracell Interference Cancellation for a DS-CDMA Synchronous Downlink with Orthogonal Codes," in Proc. VTC 2000, (Tokyo, Japan), May 2000.

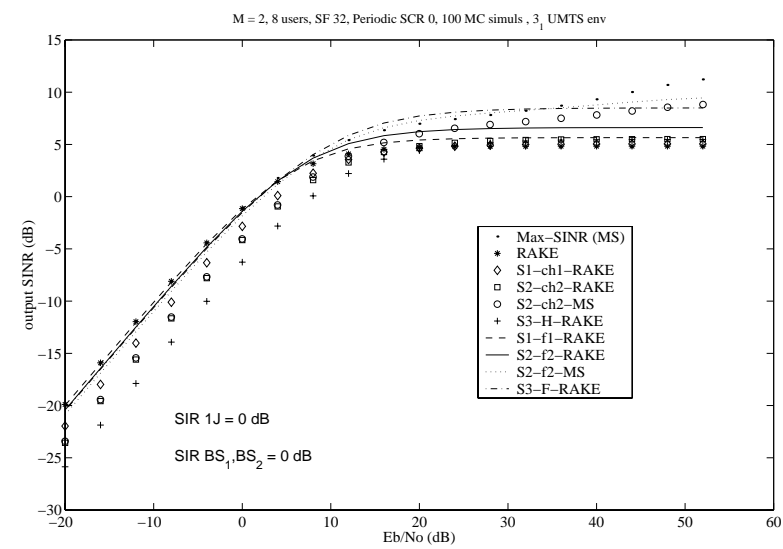

Figure 6: Output SINR versus Eb/No, 25\% loaded BSs, spreading factor 32 and equal power distribution, aperiodic scrambling, $\mathrm{ZF}$ equalizers

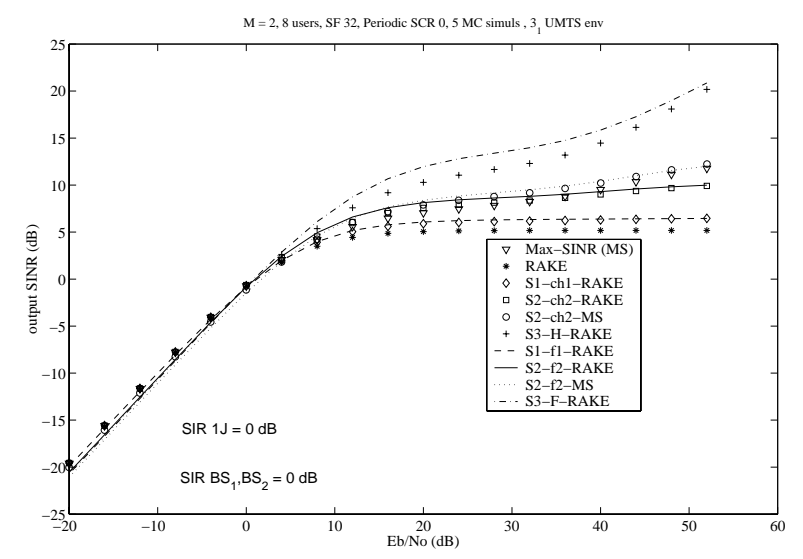

Figure 7: Output SINR versus Eb/No, 25\% loaded BSs, spreading factor 32 and equal power distribution, aperiodic scrambling, MMSE equalizers

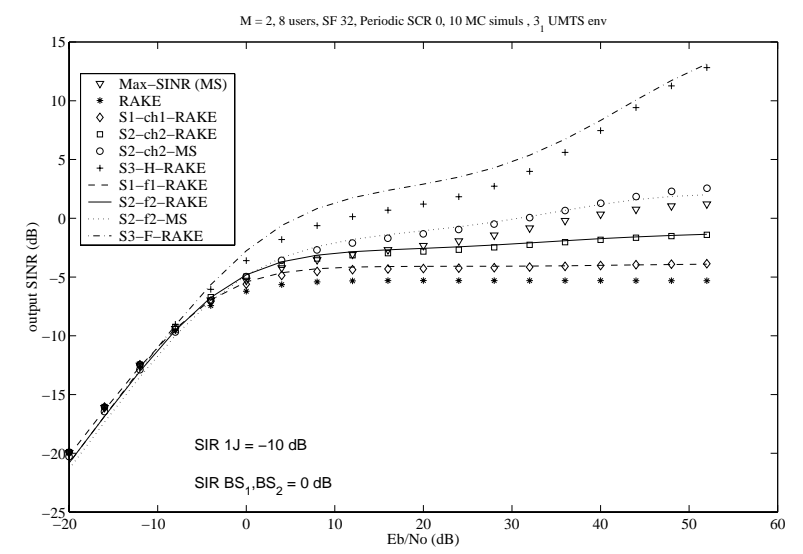

Figure 8: Output SINR versus Eb/No, 25\% loaded BSs, spreading factor 32 and near-far situation, aperiodic scrambling, MMSE equalizers 


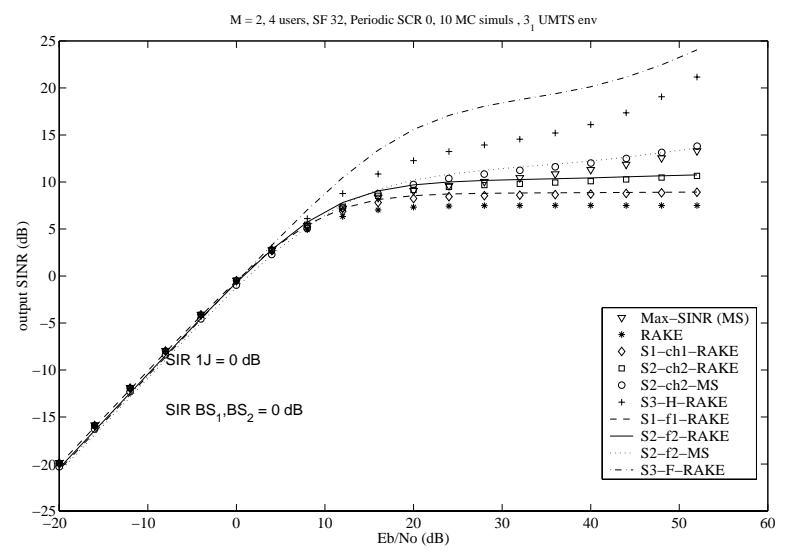

Figure 9: Output SINR versus Eb/No, $12.5 \%$ loaded BSs, spreading factor 32 and equal power distribution, aperiodic scrambling, MMSE equalizers

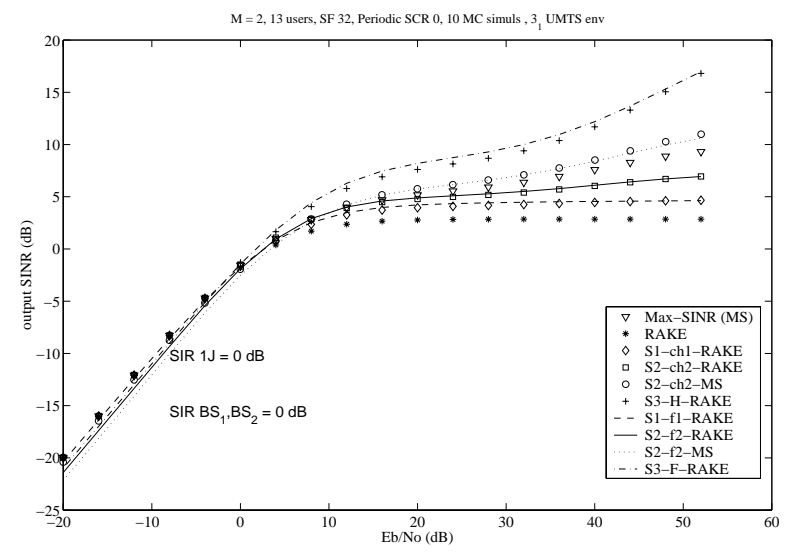

Figure 10: Output SINR versus Eb/No, 40.6\% loaded BSs, spreading factor 32 and equal power distribution, aperiodic scrambling, MMSE equalizers

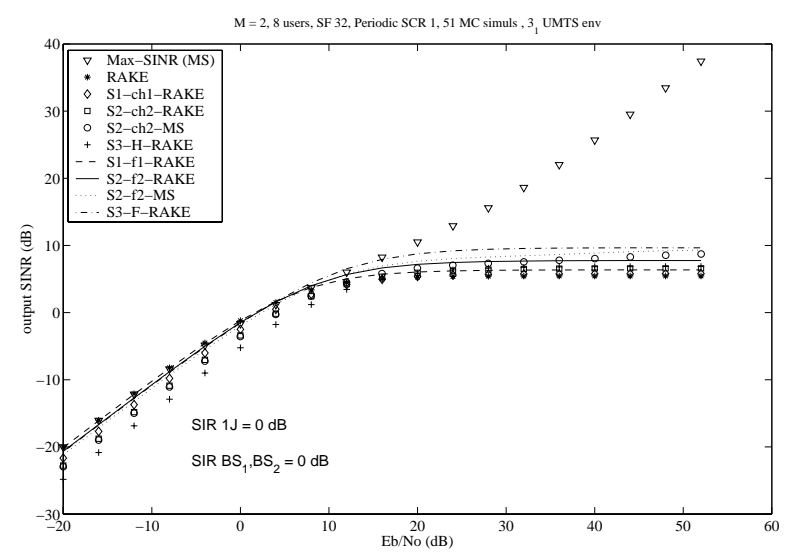

Figure 11: Output SINR versus Eb/No, 25\% loaded BSs, spreading factor 32 and equal power distribution, periodic scrambling, ZF equalizers

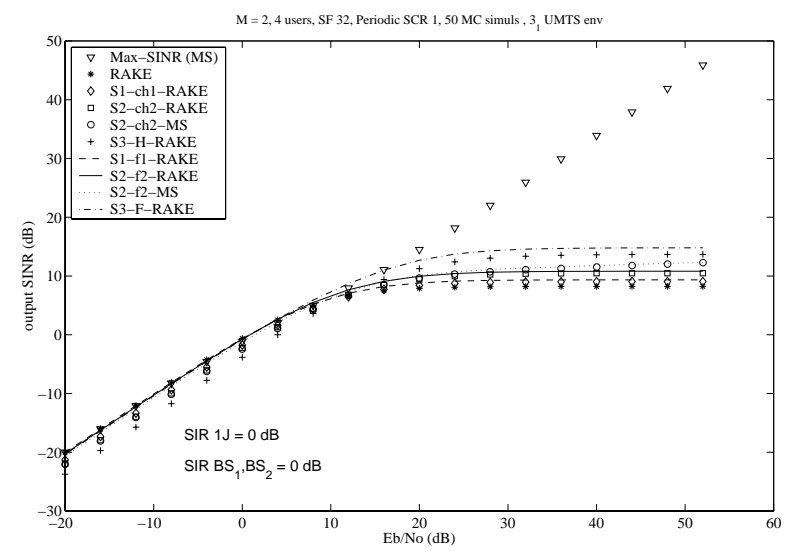

Figure 12: Output SINR versus Eb/No, 12.5\% loaded BSs, spreading factor 32 and equal power distribution, periodic scrambling, $\mathrm{ZF}$ equalizers

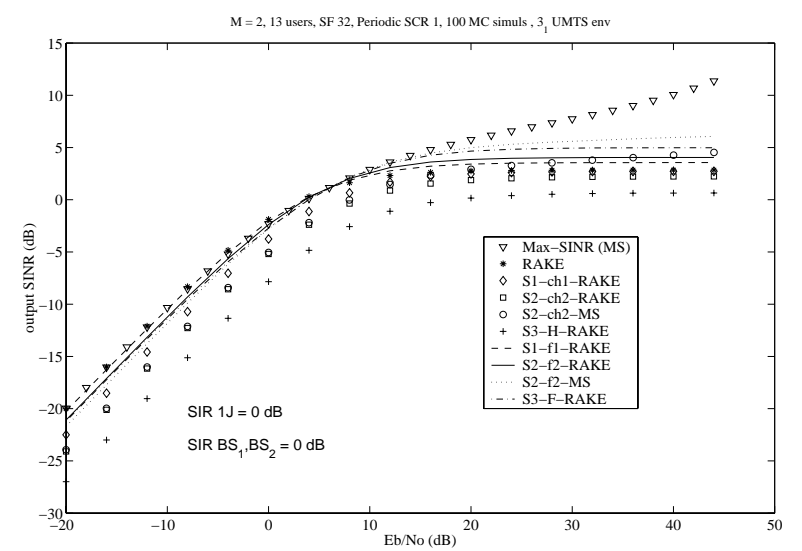

Figure 13: Output SINR versus Eb/No, 40.6\% loaded BSs, spreading factor 32 and equal power distribution, periodic scrambling, ZF equalizers

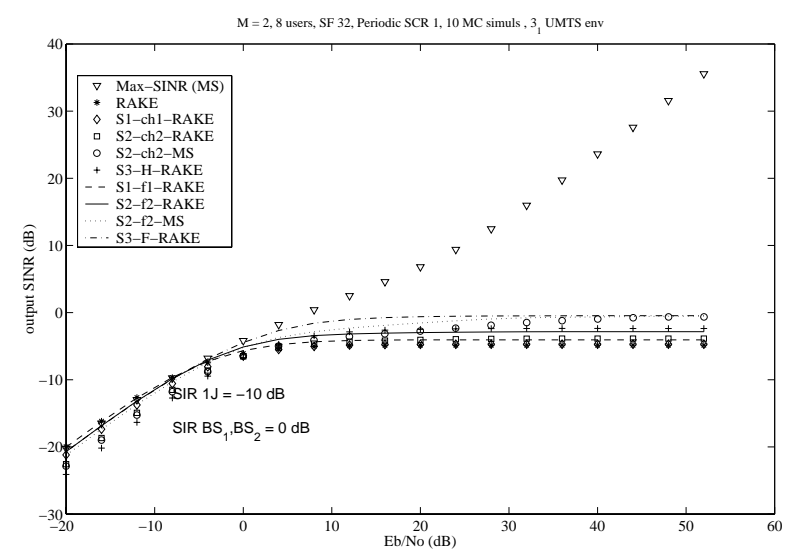

Figure 14: Output SINR versus Eb/No, 25\% loaded BSs, spreading factor 32 and near-far situation, periodic scrambling, ZF equalizers 